\title{
Somatosensory evoked blink response: findings in patients with Miller Fisher syndrome and in normal subjects
}

\author{
Hideto Miwa, Natsune Imamura, Kazunari Kogahara, Toshiko Ohori, Yoshikuni Mizuno
}

\begin{abstract}
Reflex blinking was elicited by the electrical stimulation of peripheral nerves and various parts of the body of seven of 11 patients with Miller Fisher syndrome. This reflex blinking disappeared during recovery. Reflex blinking was elicited in normal subjects only with specific stimulation of the peripheral nerves of their upper extremities. This response may be due to a release phenomenon transmitted via the brainstem reticular formation and may be useful in detecting latent CNS involvement in patients with Miller Fisher syndrome.
\end{abstract}

(F Neurol Neurosurg Psychiatry 1995;58:95-99)

Keywords: blink reflex, startle reflex, Miller Fisher syndrome, brainstem encephalitis

It has been reported that reflex blinking is evoked by multimodal sensory stimulations, such as flashes, loud sounds, tactile corneal stimulation, or electrical stimulation of the supraorbital nerve. ${ }^{12}$ These blink reflexes seem to be basic programmed responses for protecting the eyes from invasive stimuli. It is uncertain, however, whether each reflex has a common neural mechanism. We found that electrical stimulation applied to peripheral nerves or various parts of the body could directly evoke reflex blinking in patients with Miller Fisher syndrome. We made repeated observations of this characteristic phenomenon during the course of these patients' illness. Similarly, the appearance of reflex blinking elicited by peripheral nerve stimulation was also examined in normal control subjects. This characteristic blink response is referred to as the somatosensory evoked blink response (SBR) in this report.

\section{Subjects and methods}

MILLER FISHER SYNDROME

Selection criteria

Eleven patients (eight male, three female) who were admitted to the Juntendo University School of Medicine hospital during the period from October 1992 to January 1994 were diagnosed as having Miller Fisher syndrome or its related disorders. The diagnostic criteria were: (a) acute, progressive symptom development after a prodromal infection; (b) ophthalmoplegia, ataxia, or hyporeflexia without sensory or motor deficits in the corresponding limbs; and (c) complete recovery from clinical symptoms within several months. Six of the 11 patients fulfilled these criteria and were diagnosed with the complete form of the disease. The other five patients, who had numbness of extremities, obvious gaze nystagmus, or brisk deep tendon reflexes, were diagnosed as having atypical forms of the Miller Fisher syndrome.

Laboratory tests, imaging, evoked potential, and nerve conduction studies

Routine nerve conduction velocity studies were performed on all patients. Motor nerve conduction velocity studies were performed on the median, ulnar, and peroneal (or posterior tibial) nerves. Sensory conduction velocities and $F$ wave latencies in both the upper and lower extremities of patients were also obtained. Somatosensory evoked potentials (SEPs) after electrical stimulation of the median or the posterior tibial nerve and brainstem auditory evoked potentials (BAEPs) were also studied in seven patients. Computed tomography and MRI studies were performed on all patients. The CSF protein and sugar content and cell count were examined in each patient.

\section{Treatment}

All patients received steroid treatment consisting of either oral prednisolone (maximum dose $60 \mathrm{mg}$ per day) or pulse therapy with $1000 \mathrm{mg}$ of methylprednisolone for three days. Ten patients were treated with plasmapheresis.

\section{NORMAL SUBJECTS}

The control subjects were 22 men and 30 women, most of whom were medical students or staff at our university.

\section{ELECTROMYOGRAPHIC METHODS FOR}

ELICITING THE SBR

Subjects were requested to lie on a bed in a quiet room with their eyes gently closed. A pair of surface electrodes were placed bilaterally on the lateral and inferior parts of the 
Patient profiles and the result of the somatosensory evoked blink response elicited by electrical stimulation to various parts of the body

\begin{tabular}{|c|c|c|c|c|c|c|c|c|c|c|}
\hline \multirow[b]{2}{*}{ Patient } & \multirow[b]{2}{*}{ Age } & \multirow[b]{2}{*}{ Sex } & \multicolumn{4}{|c|}{ Clinical symptoms } & \multicolumn{4}{|l|}{$S B R$} \\
\hline & & & Ataxia & $D T R$ & Diplopia & Others & $M N$ & $P T N$ & $S N$ & Other sites \\
\hline \multicolumn{11}{|l|}{ Complete form: } \\
\hline 1 & 40 & $\mathbf{M}$ & + & Absent & + & & $+\star \star$ & $+\star *$ & $+*$ & \multirow{5}{*}{$\begin{array}{l}\text { Forehead }{ }^{\star} \\
\text { Forehead }^{\star}\end{array}$} \\
\hline 2 & 27 & $\mathrm{M}$ & + & Absent & + & & $+t$ & NR & NR & \\
\hline 3 & 60 & M & + & Absent & + & & $+\star \star$ & NR & NR & \\
\hline 4 & 29 & $\mathbf{M}$ & + & Absent & + & & $+{ }^{\star}$ & NR & NR & \\
\hline $\begin{array}{l}5 \\
6\end{array}$ & 50 & $\mathrm{~F}$ & + & Absent & + & & NR & NR & NR & \\
\hline \multirow{2}{*}{$\begin{array}{l}\text { Atypical form: } \\
7\end{array}$} & & & & Rosent & & & & & & \multirow{6}{*}{ 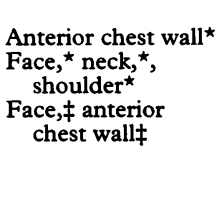 } \\
\hline & 12 & $\mathbf{M}$ & + & Brisk & + & Nystagmus & $+\star *$ & $+\star *$ & $+*$ & \\
\hline 8 & 36 & $\mathbf{M}$ & + & Brisk & + & Nystagmus & $+\ddagger$ & NR & NR & \\
\hline 9 & 65 & $\mathbf{M}$ & + & Normal & + & Nystagmus & $+\ddagger$ & NR & NR & \\
\hline 10 & 48 & M & + & Absent & + & Numbness & NR & NR & NR & \\
\hline 11 & 33 & $\mathrm{~F}$ & + & Brisk & + & Nystagmus & NR & NR & NR & \\
\hline
\end{tabular}

Somatosensory evoked blink response (SBR) is the reflex blinking elicited by electrical stimulation applied to the median nerves (MN), the posterior tibial nerves (PTN), the sural nerves (SN), and other sites of the body. $+=$ SBR could be elicited; NR $=$ no response; * Response disappeared during recovery; tresponse was still present but much less; $¥ S B R$ was not re-examined.

orbicularis oculi muscles. In some subjects, electrodes were simultaneously placed on the orbicularis oris and sternocleidomastoid muscles. Electrical stimulation, consisting of single pulses of $0.2 \mathrm{~ms}$ duration, were applied with bipolar or ring electrodes to either the supraorbital or mandibular nerve, or to peripheral nerve points including the wrist or finger (median nerve), and the ankle (posterior tibial or sural nerve). The stimulus intensity was expressed in multiples of the motor threshold (MT) or the perception threshold (PT). Electrical stimulation was applied

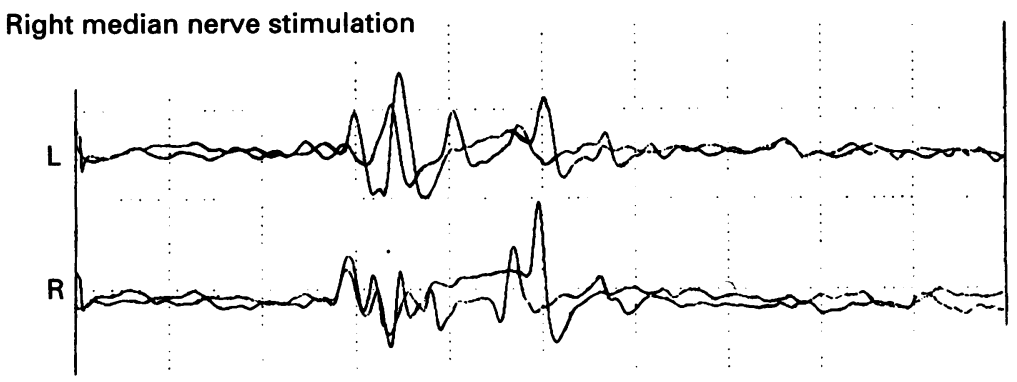

Right sural nerve stimulation

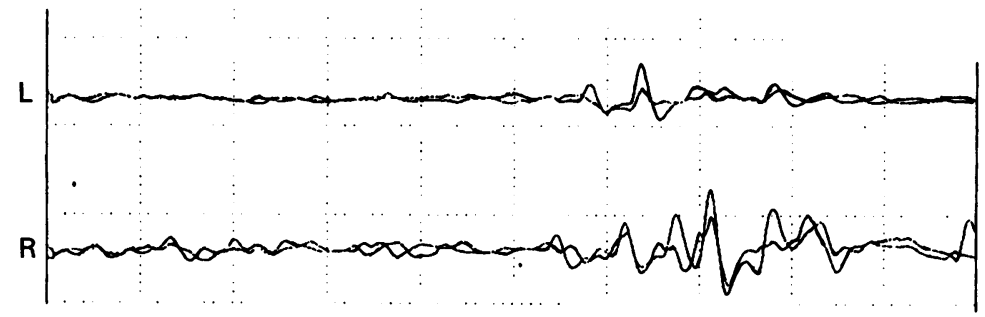

Figure 1 Representative EMGs of the somatosensory evoked blink response in patient 1; EMGs were recorded from bilateral orbicularis oculi muscles, and two traces were superimposed. The onset of sweeps of the EMGs were synchronised with the trigger pulse of electrical stimulations. The upper two traces were obtained by stimulating the left median nerve at the wrist, and the lower two were obtained by stimulating the sural nerve at the ankle. The horizontal and vertical bars indicate $20 \mathrm{~ms}$ and $100 \mu \mathrm{V}$. repeatedly at various intensities, at 10 to $15 \mathrm{~s}$ intervals, and EMG recordings were triggered simultaneously by the stimuli. The analysis time was over a period of $200 \mathrm{~ms}$. These electromyographic responses were stored on a floppy disk for later analysis. The shortest latency of the electromyographic discharges was measured on a computer screen by visual inspection. We concluded that no response was detected when the repeated stimulations, applied at least four times at intensities above 3MT, failed to elicit the SBR. In cases where SBRs were elicited by stimulating the median nerve, electrical stimulations were also applied to various parts of the body including the forehead, neck, and anterior chest wall.

The routine blink reflex elicited by supraorbital nerve stimulation was also recorded. In some control subjects, the voluntary blinking latencies in response to median nerve stimulation were also examined.

The purpose and the methods of these studies were fully explained to all those studied. Informed consent was obtained from all participants.

\section{Results}

LABORATORY TESTS, IMAGING, EVOKED

POTENTIAL AND NERVE CONDUCTION STUDIES Motor nerve conduction velocities of the patients' median, ulnar, and peroneal nerves were all normal. The sensory conduction velocities and $F$ wave latency studies performed on patients with Miller Fisher syndrome of the upper and lower extremities were also normal. The BAEP and SEP studies were performed on seven patients $(1,2,3$, $6,7,8,11)$. Latencies of N18 of the median SEPs or those of N40 of the tibial SEPs were normal. Latencies of the BAEPs were also normal. Brain CT and MRI studies were performed in all patients and were normal. The CSF showed a normal to slightly increased protein content and a normal sugar content; Pleocytosis in CSF, with 177 lymphocytes $/ \mu$, was noted in only one patient, who had an atypical syndrome. 
Left median nerve stimulation

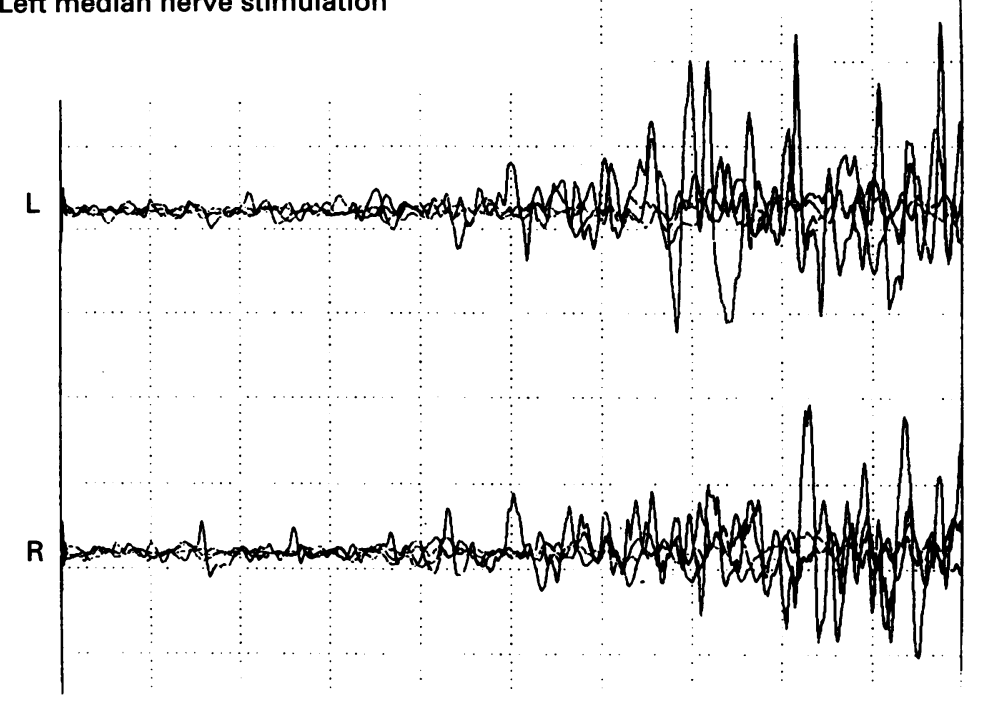

Figure 2 EMGs of the startle reaction in a normal volunteer. The EMG discharges elicited by electrical stimulations to the left median nerve were recorded from bilateral orbicularis oculi muscles in a normal subject in whom startle reactions were repeatedly induced by the stimulations. Four EMG traces were superimposed. The horizontal and vertical bars indicate $20 \mathrm{~ms}$ and $200 \mu \mathrm{V}$.

THE SOMATOSENSORY EVOKED BLINK RESPONSE IN PATIENTS WITH MILLER FISHER SYNDROME

Electrical stimulation of peripheral nerves elicited a bilateral EMG response in the orbicularis oculi muscles in seven of the 11 patients with Miller Fisher syndrome. The table summarises the clinical characteristics and the appearance of the SBRs in the SBR positive patients. The SBR was also elicited by stimulating the median nerve and the peripheral nerves of the lower extremities in two patients (fig 1). Moreover, the blinking response could also be evoked by electrical stimulation to the cutaneous surface of several body areas including the neck, shoulder, and anterior chest wall in four patients. The response was always bilateral, and although it could be elicited in the orbicularis oculi muscles, it could not be elicited in the orbicularis oris or other muscles including the sternocleidomastoid, posterior neck, and pectoralis major muscles. The threshold intensities for evoking the SBR differed among the patients. It could not be elicited by electric stimulation at intensities below $2 \mathrm{PT}$ or $(\mathrm{PT} \pm \mathrm{MT}) / 2$. The mean latencies of the SBRs elicited by stimulating the median nerves were 46.9 (SD 5.04) (range: $39 \cdot 5-54 \cdot 5) \mathrm{ms}$ for the ipsilateral and $48 \cdot 1$ (SD 4.40) (range: 40.0-52.0) ms for the contralateral nerve. The mean SBR latencies elicited by stimulating the peripheral nerves of the lower extremities were about $105 \mathrm{~ms}$ in patient 1 and $78 \mathrm{~ms}$ in patient 5 . The SBR durations varied from 20 to $100 \mathrm{~ms}$ and disappeared after repeated stimulation. It was possible to obtain stable and reproducible responses when the stimulations were applied at intervals of at least $10 \mathrm{~s}$ or more. An analysis of the relation between the appearance of the SBR and the duration of the stimulus intervals was not conducted in the present study.

The SBRs were studied repeatedly during the course of the illness in five patients at two- to four-week intervals and were found to disappear during recovery (table). In four of the five patients, the SBR disappeared before full recovery. The SBR was examined before and after three days of plasmapheresis in patient four. They had disappeared at the three day measurement. By contrast, the SBRs elicited by stimulating the upper extremities of patient 3 were still present three months from the onset of his illness. The threshold intensity for evoking the SBR was more than $3 \mathrm{MT}$, however, and the SBR was not elicited consistently.

Routine blink reflex latencies were normal for both $R 1$ and $R 2$ in all patients except patient 10 . The appearance of $\mathrm{R} 2$ was unilaterally impaired in this patient. In the patients in whom SBRs were seen, the mean latency of R1 was 10.43 (SD 0.615 ) ms. The mean latency of the ipsilateral R2 was 30.04 (SD 6.65) $\mathrm{ms}$, and that of the contralateral $\mathrm{R} 2$ was 31.98 (SD 7.01) $\mathrm{ms}$. In patients in whom SBRs were not elicited, the mean R1 latency was 10.62 (SD 1.05) $\mathrm{ms}$ and of the ipsilateral and contralateral R2 latencies were 33.02 (SD 5.27 ) $\mathrm{ms}$ and 32.76 (SD 4.88 ) $\mathrm{ms}$ respectively. The latency differences between the SBR positive and SBR negative patients are not considered here.

Electrical stimulation of the median nerve applied to the wrist or the second finger both elicited reproducible, bilateral EMG responses in the orbicularis oculi muscles in three (two men, one woman) of the 52 control subjects. Responses were always obtained bilaterally, and could be elicited in the orbicularis oculi but not the orbicularis oris muscles. The reflex discharge latencies in the ipsilateral orbicularis oculi muscle to median nerve stimulation in three control subjects were $42 \cdot 6,33 \cdot 2$, and $45 \cdot 4 \mathrm{~ms}$. Those of the contralateral side were $42 \cdot 6,34 \cdot 6$, and $50 \cdot 0$ $\mathrm{ms}$, which were equal to or longer than those of the ipsilateral side. The amplitude of the ipsilateral response was also greater than that of the contralateral side. The duration was variable (40 to $70 \mathrm{~ms}$ ) and differed among responses. Habituation was also noted. No response was evoked by stimulating the peripheral nerves of the lower extremities (the posterior tibial and the sural nerve) in any of the normal controls studied.

COMPARISON BETWEEN THE SOMATOSENSORYEVOKED BLINK RESPONSE AND THE

VOLUNTARY OR STARTLE BLINK

The latency until the onset of the electromyographic discharge in response to median nerve stimulation was around $100 \mathrm{~ms}$ or more. This latency was longer than that of the SBR. Thus the SBR was thought not to be a voluntary response but to be reflex in nature.

In some $(n=6)$ of the normal subjects, generalised startle jerks appeared during testing for SBRs. The mean latency until the 
onset of electromyographic discharges by the orbicularis oculi muscles, during a startle reaction elicited by stimulation of the median nerve, was 114.8 (SD 30.79) ms with a range of 65.8 to $160.0 \mathrm{~ms}$. The EMG bursts responsible for the startle reaction were longer than $100 \mathrm{~ms}$, and persisted beyond the time of analysis. The reaction usually appeared only once, but was repeatedly elicited in one subject (fig 2).

\section{Discussion}

Several basic and clinical studies on blink reflex have been published. ${ }^{1-5}$ To our knowledge, however, there have been no reports on reflex blinking directly induced by somatosensory stimulation.

Properties of the SBRs in patients with Miller Fisher syndrome and control subjects were as follows: (1) The SBR appeared as a phasic EMG burst in the orbicularis oculi muscles bilaterally, but its appearance was dominant on the ipsilateral side. (2) Responses were confined to the orbicularis oculi muscles, and did not include other muscles, such as the orbicularis oris, sternocleidomastoid, or pectoralis major muscles. (3) The latencies to the onset of the SBR elicited by median nerve stimulation ranged from 40 to $50 \mathrm{~ms}$. The SBRs elicited by stimulating peripheral nerves of the lower extremities had longer latencies. (4) Habituation occurred after repeated stimulation. (5) The SBR could be elicited not only by the direct stimulation of peripheral nerves but also by stimulating various cutaneous body areas in patients with Miller Fisher syndrome. In normal subjects, SBRs were elicited only with stimulation to the peripheral nerves of the upper extremities. It is presumed that the SBR is not a result of alterations in central neural connections but a release phenomenon, because the SBR could be evoked in a few normal subjects. One important difference between the SBR in normal subjects and in patients with Miller Fisher syndrome is how much wider an area can evoke an SBR in the patients.

\section{PATHOPHYSIOLOGY OF THE SOMATOSENSORY} EVOKED BLINK RESPONSE

It is not easy to speculate on which exact neural pathways subserve the SBR. The peripheral afferent pathway is possibly of cutaneous origin, because the response can be elicited by stimulating the cutaneous surfaces of the anterior chest wall or neck, or the sural nerve. Similar responses can also be elicited by stimulating the median nerve at the second finger or the wrist. The presence of habituation may indicate that the central pathways are polysynaptic. The final output must be mediated by motor neurons in the facial nerve nucleus responsible for blinking; however, the central pathways to the facial nerve nucleus are uncertain. It has been shown that spinal inputs to the facial nucleus exist in cats. ${ }^{6}$ As blink reflexes, induced by both supraorbital nerve or auditory stimulation, are transmitted via the brainstem reticular formation, ${ }^{347}$ it seems likely that the brainstem reticular formation also plays a part in generating SBRs. This speculation may be supported by anatomical findings that the brainstem reticular formation receives rich, cutaneous inputs from diverse parts of the body and has polysynaptic connections with the facial nerve nuclei. ${ }^{89}$ Presently, it is unclear which neural structures are responsible for the abnormal sensory gating between somatosensory inputs and motor outflow of the facial nucleus.

It seems important to compare the SBR with the $R 2$ response of the blink reflex, because the SBR shares common characteristics with the $\mathrm{R} 2$ response. In both cases, the response is confined to the orbicularis oculi muscles (possibly of cutaneous origin), and is easily habituated. It also may be postulated that the SBR is a variation of the $R 2$ response induced by an abnormal widening of the area that can evoke the $R 2$ response. Although a recent study has shown that the $R 2$ response of the blink reflex is very depressed by the delivery of a conditioning stimulus to the limbs, ${ }^{10}$ it is still uncertain whether the extratrigeminal sensory input has a latent and direct connection to the blink reflex pathways.

A relation between the SBR and the startle reaction should also be considered because blinking is the most consistent and easily elicitable component of the startle reaction. Two of our findings in the control subjects show how the SBR differs from the startle reaction. Firstly, the duration and latency of the EMG response for the startle reaction were variable and much longer than those for the SBR. Secondly, the startle reaction led to generalised jerks, not confined to the orbicularis oculi muscles. The possibility that the SBR constitutes an initial part of the startle reaction in the absence of a subsequent generalised startle response, however, should not be fully excluded. Further studies are needed to determine whether the SBR should be classified as a type of abnormally enhanced R2 like response or a variation of the startle blink.

THE PATHOLOGICAL SIGNIFICANCE OF THE SOMATOSENSORY EVOKED BLINK RESPONSE Another important finding in the present study was that SBRs disappeared during a patient's recovery from Miller Fisher syndrome. The pathogenesis and the anatomical substrate of Miller Fisher syndrome remain uncertain. One possibility is that it is a variant of GuillainBarre syndrome. ${ }^{11}$ Another is that the syndrome is a type of brainstem encephalitis. ${ }^{12}$ It is interesting to know that, in Guillain-Barre syndrome, the $R 1$ response of the blink reflex is absent or delayed and that the Miller Fisher syndrome, on the other hand, does not regularly affect the blink reflex. ${ }^{5}$ The finding that SBRs disappeared during the course of a patient's illness suggests that a certain pathological CNS condition existed in patients with Miller Fisher syndrome. 
Finally, the SBR can be recorded easily when it is present, with the same technique as for a routine blink reflex. We propose that the SBR is useful for detecting latent CNS involvement in these types of disorders.

1 Rushworth G. Observations on blink reflexes. $f$ Neurol Neurosurg Psychiatry 1962;25:93-108.

2 Yates SK, Brown WF. Light-stimulus-evoked blink reflex: methods, normal values, relation to other blink reflexes, and observations in multiple sclerosis. Neurology 1981; 31:272-81.

3 Kimura J. The blink reflex as a test for brainstem and higher nervous system function. In: Desmedt JE eds. New developments in electromyography and clinical electropew developments in electromyography and clinic

4 Ongerboer de Visser BW, Kuypers HGJM: Late blink reflex changes in lateral medullary lesions. Brain 1978; 101:285-94.
5 Kimura J. Electrodiagnosis in disease of nerve and muscle. Principles and practice. Philadelphia: FA Davis Co, 1989: Principles and practice. Philadelphia: FA Davis Co, 1989:

Tanaka T, Yu H, Kitai ST. Trigeminal and spinal inputs to the facial nucleus. Brain Res 1971;33:504-8.

7 Hori A, Yasuhara A, Naito H, et al. Blink reflex elicited by auditory stimulation in the rabbit. F-Neurol Sci 1986; 76:49-59.

8 Segundo JP, Takenaka T, Encabo H. Somatic sensory properties of bulbar reticular neurons. $\mathcal{F}$ Neurophysiol 1967;30:1220-38.

9 Hirrichesen CFL, Watoson CD. Brainstem projections to the facial nucleus of the rat. Brain Behav Evol 1983;22: 153-63.

10 Rossi A, Scarpini C. Gating of trigemino-facial reflex from low-threshold treigeminal and extratrigeminal cutaneous fibres in humans. F Neurol Neurosurg Psychiatry 1992;55: 774-80.

11 Fisher $M$. An unusual variant of acute idiopathic polyneuritis (syndrome of ophthalmoplegia, ataxia and areneuritis (syndrome of ophthalmoplegia,

12 Al-Din AN, Anderson M, Bickerstaff ER, et al. Brainstem encephalitis and the syndrome of Miller Fisher: a clinical study. Brain 1982;105:481-95. 